\title{
El ingreso de la gran mayoría (IGM): Una nueva medida de la desigualdad global entre países
}

\author{
Anwar Shaikh* y Amr Ragab**
}

\section{RESUMEN}

Este artículo hace una reflexión crítica sobre la pertinencia de emplear indicadores económicos, como el PIB por habitante y el índice de desarrollo humano, para realizar mediciones comparativas de desarrollo entre países. Los autores proponen un nuevo indicador: el ingreso de la gran mayoría (IGM), que combina ingreso con desigualdad a partir de un ejercicio empírico de análisis de desarrollo comparativo entre países. Los resultados obtenidos a partir de este indicador contrastan con aquellos obtenidos con el ingreso nacional neto por habitante (INNph). Se comprueba que las medidas de ingreso promedio no constituyen buenas aproximaciones de los ingresos de las grandes mayorías. Se formulan asimismo propuestas de política y señalan algunos de los aspectos de este tema que forman parte de la agenda de investigaciones futuras.

Palabras clave: análisis de desarrollo comparativo, mediciones entre países, ingreso de la gran mayoría (IGM).

Clasificación JEL: E01.

\begin{abstract}
The aim of this article is to do a critical reflection about de use of economical indicators, as PIB per capita and human development index (HDI), in order to make development comparative measurement across countries. The authors propose a new indicator: the Vast Majority Income (VMI), which shares income with inequality by doing an empirical analysis of comparative development among countries. The outcomes got through this last indicator contrast with those coming from Net National Income per capita. They test and find out that measures of average income are not good instruments to study the vast majority income. Finally, there are some political proposals and show some issues that must be undertaken in the future.
\end{abstract}

Key words: new comparative development, measurement across countries, vast majority indicator.

Classification JEL: E01.

* Profesor del Departamento de Economía en The New School for Social Research e investigador en el Schwartz Center for Economic Policy Analysis (SCEPA).

**Asistente de investigación en el SCEPA. Esta nota se basa en el trabajo "An international comparison of the incomes of the Vast Majority", Working Paper 2007-3, SCEPA disponible en www. newschool.edu/scepa. Traducción de José Luis Estrada López. 


\section{LAS MEDIDAS DE INGRESO Y DESIGUALDAD EN EL ÁMBITO MUNDIAL}

\section{INTRODUCCIÓN}

El PIB por habitante es la medida más popular de los niveles internacionales de desarrollo. Es muy clara y ampliamente disponible entre países y en el tiempo (Frumkin, 2000). También es aceptado que esta medida es un indicador indirecto imperfecto para factores importantes tales como salud, educación y el bienestar en general (Cowen, 2007). En consecuencia, una alternativa ha sido construir un índice compuesto, como el índice de desarrollo humano (IDH) del Programa de las Naciones Unidas para el Desarrollo (UNDP, por sus siglas en inglés), a que combina el ingreso por habitante con la esperanza de vida y la escolaridad, (1990). Este índice, sin embargo, es difícil de compilar y está disponible únicamente para años recientes, además de que, por su naturaleza de índice, no puede expresar el estándar de vida de la población subyacente; lo único que puede ofrecer son las posiciones relativas de las naciones en cada momento y los cambios correspondientes en el tiempo. En todo caso, resulta que el ranking mediante el ingreso por habitante y el IDH están sumamente correlacionados (Kelley, 1991).

Puesto que el PIB por habitante sí ofrece una medida absoluta del ingreso, es razonable que siga manteniendo su popularidad. Pero, tanto el PIB por habitante como el índice de desarrollo humano padecen el que "son promedios que esconden amplias disparidades entre la población" (Kelley, 1991). En consecuencia, se torna necesario complementar estas medidas, por un lado, con información sobre la desigualdad distributiva (como el coeficiente Gini) y, por el otro ajustar directamente la medida de ingreso por habitante y otras variables por las variaciones distributivas.

\section{EL INGRESO DE LA GRAN MAYORÍA: COMBINANDO INGRESO CON DESIGUALDAD}

Si bien el PIB por habitante tiene la gran virtud de ser una medida absoluta del ingreso nacional promedio, el hecho de que la distribución del ingreso y del consumo pueda presentar grandes sesgos al interior de los países hace que no se le pueda interpretar como el ingreso de la gran mayoría de la población. Esto es en especial verdadero en el mundo en desarrollo, donde un aumento en el ingreso por habitante puede acompañarse de un empeoramiento en la distribución del 
ingreso, de manera que el nivel de vida de la gran mayoría de la población puede declinar a pesar del alza en el ingreso por habitante.

Considérese un ejemplo en el cual hay cinco personas con ingresos de 5, $10,15,20$ y 50 dólares respectivamente. El ingreso por habitante de la gran mayoría (es decir, del primer $80 \%$ de la población) es el promedio de los primeros cuatro ingresos, el cual asciende a 12.5 dólares por persona. En contraste, el promedio global es de 20. Su relación es de 0.625 (=12.5/20), lo que nos revela que, con la información actual, el ingreso por habitante será un indicador indirecto deficiente del ingreso de la gran mayoría (IGM). Además, si dicha relación varía en el tiempo, la tendencia del ingreso por habitante también será una guía poco confiable del progreso del IGM.

Lo que se necesita, por tanto, es una medida directa del nivel de vida de la gran mayoría. En el trabajo que sirve de base para esta nota desarrollamos una nueva medida que llamamos el ingreso de la gran mayoría, la cual representa el promedio de ingreso de $80 \%$ de la población con ingresos más bajos. La información disponible sobre la distribución del ingreso nos permite calcular directamente la relación del IGM con el promedio. Al multiplicar este cociente por una medida de ingreso promedio por habitante se obtiene el nivel del IGM. ${ }^{1}$

\section{VARIACIONES INTERNACIONALES EN LOS INGRESOS DE LA GRAN MAYORÍA (IGM)}

La gráfica 1 muestra que los ingresos reales ICM en 2000 comprenden un amplio rango: en cifras redondeadas, hacia un extremo de la escala se encuentra Luxemburgo (30 000 dólares), Noruega (22 000 dólares) y Estados Unidos (21 000 dólares). En el otro extremo se encuentran Etiopía (500 dólares) y Camboya (300 dólares). En este cuadro los países están clasificados de mayor a menor de acuerdo con su ingreso nacional neto por habitante (INNph); ${ }^{2}$ se observa que Luxem-

\footnotetext{
${ }^{1}$ La información sobre distribución proviene de la base de datos sobre la desigualdad del ingreso en el mundo, publicada por la Universidad de las Naciones Unidas y el Instituto Mundial para la Investigación sobre Desarrollo Económico. Esta información es muy variada y la cobertura temporal es variable para los primeros años y para la mayoría de los países que no son miembros de la OCDE. En el trabajo se emplea el mayor subconjunto de datos que fue posible construir de manera congruente (643 observaciones) para el indicador de la distribución del ingreso personal disponible.

${ }^{2}$ Usamos el ingreso nacional neto por habitante, en lugar de PIB por habitante como la medida apropiada para promediar el ingreso nacional por habitante, ya que la primera incluye el ingreso factorial que procede del resto del mundo, a la vez que excluye la depreciación (la cual no debe entrar en la medida del ingreso personal); para mayores detalles consultar el artículo original citado en la nota 1 .
} 
122 ECONOMÍA: TEORÍA Y PRÁCTICA • Nueva Época, número 31, julio-diciembre 2009

burgo está en primer lugar, Estados Unidos en segundo, Noruega en tercero y así sucesivamente.

Gráfica 1. Ingreso de la mayoría (IMG) real por habitante, entre países (los ingresos se convirtieron a dólares de Estados Unidos mediante los tipos de cambio de la PPA, 2000)

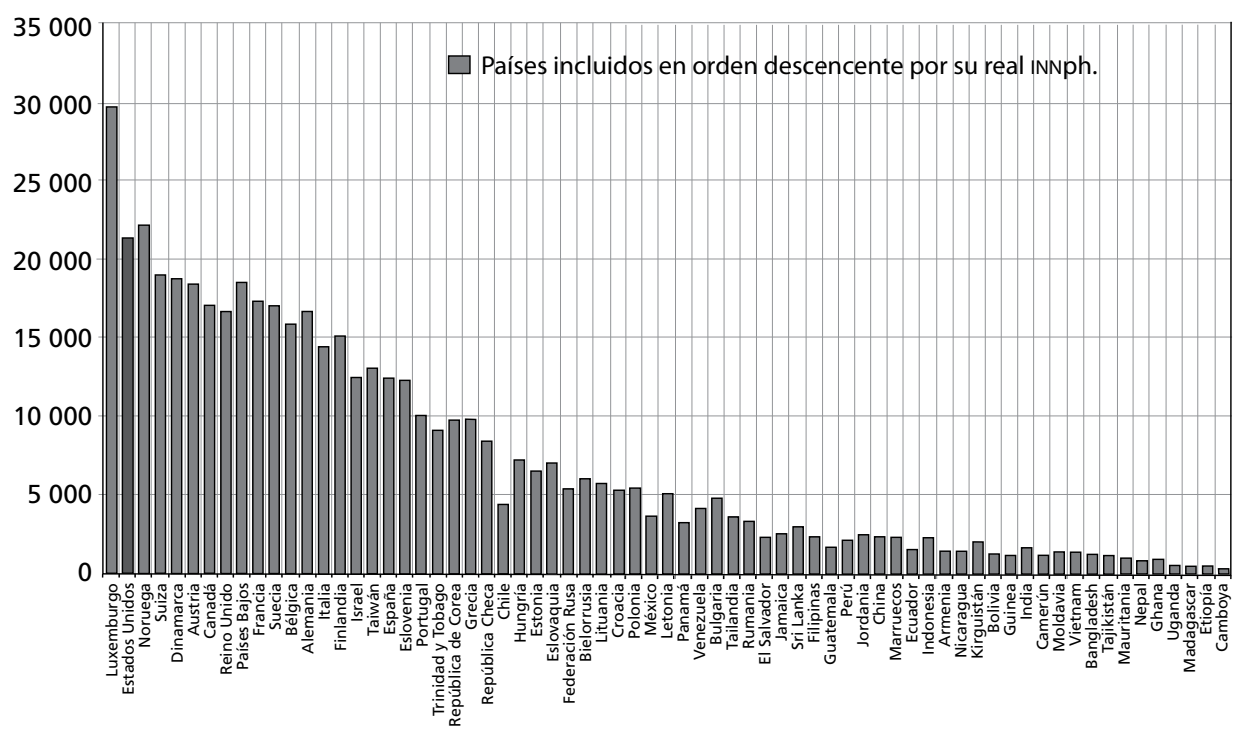

Gráfica 2. Cambio de rango, utilizando IGM en lugar de ingreso nacional neto

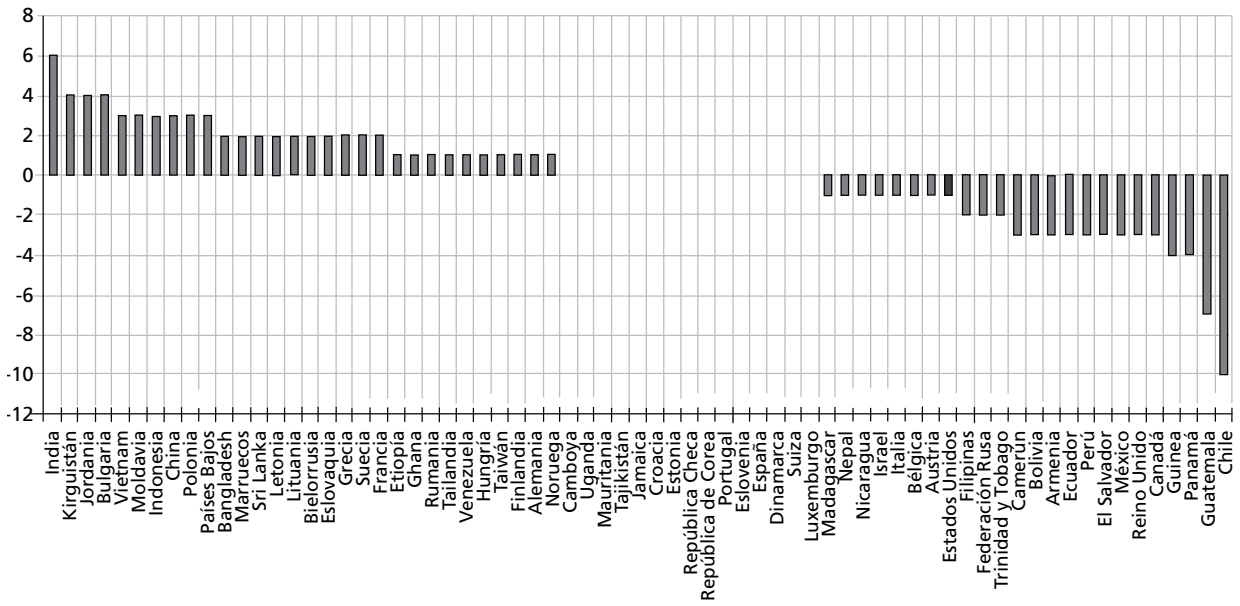


Cuando se pone atención al IGM en lugar del ingreso nacional neto por habitante, la representación cambia significativamente. En el cuadro 2 se puede apreciar que el IGM de Noruega es mayor al de Estados Unidos, por lo que, en términos de este nuevo indicador, Noruega se desplaza al segundo lugar, mientras que Estados Unidos baja al tercero. Esto se debe a que la desigualdad en el ingreso es considerablemente mayor en Estados Unidos que en Noruega. Por otra parte, Chile ofrece un ejemplo aún más llamativo de los efectos negativos de la desigualdad: en términos de la medida convencional de ingreso nacional neto por habitante, Chile está en una posición similar a Hungría, pero de acuerdo con el IGM su posición es similar a la de Venezuela.

El cuadro 1 muestra el IGM, el ingreso nacional neto por habitante y una medida del ingreso por habitante relativo del $20 \%$ más rico de la población, a la cual denominamos el ingreso de la minoría opulenta (IMO). También se presenta el ranking de los países de acuerdo con el ingreso nacional neto por habitante y el correspondiente al IGM, así como la diferencia entre ambos, la cual se registra en la última columna. Los países se han enumerado de acuerdo con esta diferencia. Se puede observar que India aparece en el lugar más alto de la lista debido a que asciende seis lugares; Jordania y Bulgaria ascienden cuatro lugares, cada uno, mientras que Vietnam, Países Bajos y China ascienden cada uno tres lugares. En el otro extremo, México, Reino Unido y Canadá descienden cada uno tres lugares. ${ }^{3}$ Por otra parte, Panamá desciende cuatro lugares, Guatemala desciende siete en tanto Chile aparece al final de la lista, cayendo 10 lugares.

\section{IMPLICACIONES DE POLÍTICA}

Es usual tratar los niveles de ingreso y los de desigualdad en forma separada; el primero, mediante un promedio por habitante de medidas de ingreso, tales como el PIB por habitante, y el segundo mediante medidas de desigualdad, como el coeficiente Gini. Nuestra investigación demuestra que el ingreso por habitante de una fracción de la población combina estos dos aspectos de un modo intuitivamente útil, siendo en especial interesante el ingreso de la gran mayoría real por habitante de una nación. La dimensión y evolución temporal de esta medida (IGM) posee gran importancia para las democracias modernas.

\footnotetext{
${ }^{3}$ Por ejemplo, Canadá se encuentra en el lugar séptimo en la escala mundial en términos del ingreso nacional neto por habitante, pero en el décimo mundial de acuerdo con el IGM. Es decir, desciendió tres lugares al pasar de uno a otro indicador.
} 
124 ECONOMÍA: TEORÍA Y PRÁCTICA • Nueva Época, número 31, julio-diciembre 2009

Cuadro 1. IGM per cápita, INNph y lugar de países por cada medida

\begin{tabular}{|c|c|c|c|c|c|c|c|}
\hline \multirow[b]{2}{*}{ País } & \multirow[b]{2}{*}{$\begin{array}{l}\text { Coeficiente } \\
\text { Gini }\end{array}$} & \multicolumn{3}{|c|}{$\begin{array}{l}\text { En términos reales, por habitante } \\
\text { (dólares internacionales) }\end{array}$} & \multicolumn{2}{|c|}{ Lugar } & \multirow[b]{2}{*}{$\begin{array}{l}\text { Diferencia } \\
\text { de lugar }\end{array}$} \\
\hline & & $\begin{array}{c}\text { Ingreso } \\
\text { nacional neto } \\
\text { por habitante } \\
\text { (INNph) }\end{array}$ & $\begin{array}{c}\text { Ingreso de la } \\
\text { gran mayoría } \\
\text { (IGM) }\end{array}$ & $\begin{array}{c}\text { Ingreso de la } \\
\text { minoría } \\
\text { opulenta } \\
\text { (IMO) }\end{array}$ & (INNph) & (IGM) & \\
\hline India & 36.00 & $\$ 2371$ & $\$ 1651$ & $\$ 5247$ & 57 & 51 & 6 \\
\hline Kirguistán & 37.00 & $\$ 2886$ & $\$ 2039$ & $\$ 6275$ & 54 & 50 & 4 \\
\hline Jordania & 36.30 & $\$ 3526$ & $\$ 2449$ & $\$ 7833$ & 47 & 43 & 4 \\
\hline Bulgaria & 30.70 & $\$ 6282$ & $\$ 4809$ & $\$ 12175$ & 38 & 34 & 4 \\
\hline Vietnam & 37.30 & $\$ 1993$ & $\$ 1359$ & $\$ 4531$ & 60 & 57 & 3 \\
\hline Moldavia & 39.55 & $\$ 2021$ & $\$ 1379$ & $\$ 4590$ & 59 & 56 & 3 \\
\hline Indonesia & 36.50 & $\$ 3308$ & $\$ 2277$ & $\$ 7434$ & 51 & 48 & 3 \\
\hline China & 40.30 & $\$ 3478$ & $\$ 2320$ & $\$ 8111$ & 48 & 45 & 3 \\
\hline Polonia & 32.45 & $\$ 7228$ & $\$ 5390$ & $\$ 14579$ & 33 & 30 & 3 \\
\hline Países Bajos & 25.50 & $\$ 22404$ & $\$ 18483$ & $\$ 38087$ & 9 & 6 & 3 \\
\hline Bangladesh & 35.85 & $\$ 1768$ & $\$ 1227$ & $\$ 3930$ & 61 & 59 & 2 \\
\hline Marruecos & 39.20 & $\$ 3436$ & $\$ 2306$ & $\$ 7954$ & 49 & 47 & 2 \\
\hline Sri Lanka & 27.60 & $\$ 3767$ & $\$ 2965$ & $\$ 6971$ & 43 & 41 & 2 \\
\hline Letonia & 34.30 & $\$ 7034$ & $\$ 5100$ & $\$ 14772$ & 35 & 33 & 2 \\
\hline Lituania & 33.00 & $\$ 7742$ & $\$ 5702$ & $\$ 15904$ & 31 & 29 & 2 \\
\hline Bielorrusia & 30.75 & $\$ 7979$ & $\$ 6035$ & $\$ 15755$ & 30 & 28 & 2 \\
\hline $\begin{array}{l}\text { República } \\
\text { Eslovaca }\end{array}$ & 26.15 & $\$ 8651$ & $\$ 6999$ & $\$ 15262$ & 28 & 26 & 2 \\
\hline Grecia & 32.30 & $\$ 12847$ & $\$ 9796$ & $\$ 25052$ & 23 & 21 & 2 \\
\hline Suecia & 28.20 & $\$ 21892$ & $\$ 17023$ & $\$ 41369$ & 11 & 9 & 2 \\
\hline Francia & 28.20 & $\$ 22248$ & $\$ 17242$ & $\$ 42271$ & 10 & 8 & 2 \\
\hline Etiopía & 36.15 & $\$ 697$ & $\$ 484$ & $\$ 1546$ & 68 & 67 & 1 \\
\hline Ghana & 33.90 & $\$ 1290$ & $\$ 932$ & $\$ 2723$ & 65 & 64 & 1 \\
\hline Rumania & 29.85 & $\$ 4374$ & $\$ 3358$ & $\$ 8437$ & 40 & 39 & 1 \\
\hline Tailandia & 44.60 & $\$ 5893$ & $\$ 3630$ & $\$ 14944$ & 39 & 38 & 1 \\
\hline Venezuela & 45.80 & $\$ 6666$ & $\$ 4113$ & $\$ 16878$ & 37 & 36 & 1 \\
\hline Hungría & 30.30 & $\$ 9464$ & $\$ 7216$ & $\$ 18455$ & 26 & 25 & 1 \\
\hline Taiwán & 31.55 & $\$ 17463$ & $\$ 13059$ & $\$ 35083$ & 17 & 16 & 1 \\
\hline Finlandia & 25.98 & $\$ 18754$ & $\$ 15069$ & $\$ 33490$ & 15 & 14 & 1 \\
\hline Alemania & 27.60 & $\$ 21078$ & $\$ 16641$ & $\$ 38825$ & 13 & 12 & 1 \\
\hline Noruega & 27.40 & $\$ 28153$ & $\$ 22092$ & $\$ 52394$ & 3 & 2 & 1 \\
\hline Camboya & 44.50 & $\$ 494$ & $\$ 295$ & $\$ 1288$ & 69 & 69 & 0 \\
\hline Uganda & 46.90 & $\$ 963$ & $\$ 559$ & $\$ 2580$ & 66 & 66 & 0 \\
\hline Mauritania & 38.90 & $\$ 1432$ & $\$ 974$ & $\$ 3263$ & 63 & 63 & 0 \\
\hline Tajikistán & 33.30 & $\$ 1511$ & $\$ 1104$ & $\$ 3142$ & 62 & 62 & 0 \\
\hline
\end{tabular}


Cuadro 1. IGM per cápita, INNph y lugar de países por cada medida (Conclusión.)

\begin{tabular}{|c|c|c|c|c|c|c|c|}
\hline \multirow{2}{*}{\begin{tabular}{|l} 
\\
País
\end{tabular}} & \multirow[b]{2}{*}{$\begin{array}{l}\text { Coeficiente } \\
\text { Gini }\end{array}$} & \multicolumn{3}{|c|}{$\begin{array}{c}\text { En términos reales, por habitante } \\
\text { (dólares internacionales) }\end{array}$} & \multicolumn{2}{|c|}{ Lugar } & \multirow[b]{2}{*}{$\begin{array}{c}\text { Diferencia } \\
\text { de lugar }\end{array}$} \\
\hline & & $\begin{array}{c}\text { Ingreso } \\
\text { nacional } \\
\text { neto por } \\
\text { habitante } \\
\text { (INNph) }\end{array}$ & $\begin{array}{l}\text { Ingreso de } \\
\text { la gran } \\
\text { mayoría } \\
(\text { IGM) }\end{array}$ & $\begin{array}{l}\text { Ingreso de } \\
\text { la minoría } \\
\text { opulenta } \\
\text { (IMO) }\end{array}$ & INNph & IGM & \\
\hline Jamaica & 43.30 & $\$ 4013$ & $\$ 2528$ & $\$ 9953$ & 42 & 42 & 0 \\
\hline Croacia & 33,95 & $\$ 7278$ & $\$ 5310$ & $\$ 15154$ & 32 & 32 & 0 \\
\hline Estonia & 36.50 & $\$ 9227$ & $\$ 6489$ & $\$ 20178$ & 27 & 27 & 0 \\
\hline Rep. Checa & 25.90 & $\$ 10487$ & $\$ 8370$ & $\$ 18956$ & 24 & 24 & 0 \\
\hline Rep. de Corea & 36.90 & $\$ 13371$ & $\$ 9765$ & $\$ 27792$ & 22 & 22 & 0 \\
\hline Portugal & 34.70 & $\$ 13894$ & $\$ 10073$ & $\$ 29178$ & 20 & 20 & 0 \\
\hline Eslovenia & 25.15 & $\$ 15079$ & $\$ 12267$ & $\$ 26329$ & 19 & 19 & 0 \\
\hline España & 32.48 & $\$ 16694$ & $\$ 12370$ & $\$ 33989$ & 18 & 18 & 0 \\
\hline Dinamarca & 24.85 & $\$ 22900$ & $\$ 18702$ & $\$ 39696$ & 5 & 5 & 0 \\
\hline Suiza & 35.90 & $\$ 26246$ & $\$ 18949$ & $\$ 55431$ & 4 & 4 & 0 \\
\hline Luxemburgo & 28.25 & $\$ 37736$ & $\$ 29560$ & $\$ 70438$ & 1 & 1 & 0 \\
\hline Madagascar & 48.50 & $\$ 814$ & $\$ 464$ & $\$ 2.214$ & 67 & 68 & -1 \\
\hline Nepal & 42.55 & $\$ 1337$ & $\$ 832$ & $\$ 3357$ & 64 & 65 & -1 \\
\hline Nicaragua & 55.50 & $\$ 2913$ & $\$ 1478$ & $\$ 8651$ & 53 & 54 & -1 \\
\hline Israel & 38.05 & $\$ 17779$ & $\$ 12405$ & $\$ 39277$ & 16 & 17 & -1 \\
\hline Italia & 33.80 & $\$ 19366$ & $\$ 14407$ & $\$ 39203$ & 14 & 15 & -1 \\
\hline Bélgica & 31.33 & $\$ 21381$ & $\$ 15804$ & $\$ 43689$ & 12 & 13 & -1 \\
\hline Austria & 26.45 & $\$ 22733$ & $\$ 18362$ & $\$ 40217$ & 6 & 7 & -1 \\
\hline EUA & 39.75 & $\$ 31283$ & $\$ 21309$ & $\$ 71178$ & 2 & 3 & -1 \\
\hline Filipinas & 44.15 & $\$ 3752$ & $\$ 2316$ & $\$ 9496$ & 44 & 46 & -2 \\
\hline Fed. Rusa & 42.50 & $\$ 8265$ & $\$ 5374$ & $\$ 19825$ & 29 & 31 & -2 \\
\hline Trin y Tob & 40.20 & $\$ 13445$ & $\$ 9094$ & $\$ 30850$ & 21 & 23 & -2 \\
\hline Camerún & 50.80 & $\$ 2145$ & $\$ 1193$ & $\$ 5952$ & 58 & 61 & -3 \\
\hline Bolivia & 58.05 & $\$ 2642$ & $\$ 1273$ & $\$ 8118$ & 55 & 58 & -3 \\
\hline Armenia & 56.05 & $\$ 2923$ & $\$ 1443$ & $\$ 8844$ & 52 & 55 & -3 \\
\hline Ecuador & 58.80 & $\$ 3310$ & $\$ 1548$ & $\$ 10354$ & 50 & 53 & -3 \\
\hline Perú & 46.50 & $\$ 3553$ & $\$ 2115$ & $\$ 9307$ & 46 & 49 & -3 \\
\hline El Salvador & 53.45 & $\$ 4372$ & $\$ 2333$ & $\$ 12526$ & 41 & 44 & -3 \\
\hline México & 54.20 & $\$ 7115$ & $\$ 3653$ & $\$ 20962$ & 34 & 37 & -3 \\
\hline Reino Unido & 33.05 & $\$ 22454$ & $\$ 16645$ & $\$ 45689$ & 8 & 11 & -3 \\
\hline Canadá & 32.40 & $\$ 22655$ & $\$ 17021$ & $\$ 45192$ & 7 & 10 & -3 \\
\hline Guinea & 55.10 & $\$ 2384$ & $\$ 1200$ & $\$ 7122$ & 56 & 60 & -4 \\
\hline Panamá & 57.80 & $\$ 6728$ & $\$ 3236$ & $\$ 20696$ & 36 & 40 & -4 \\
\hline Guatemala & 59.80 & $\$ 3613$ & $\$ 1631$ & $\$ 11547$ & 45 & 52 & -7 \\
\hline Chile & 58.20 & $\$ 9512$ & $\$ 4371$ & $\$ 30077$ & 25 & 35 & -10 \\
\hline
\end{tabular}

Fuente: "An International Comparison of the Incomes of the Vast Majority", SCEPA Working Paper, 2007-3, disponible en www.newschool.edu/cepa. 
Puesto que hay una gran variabilidad internacional de la relación entre el IGM y el ingreso por habitante, las medidas de ingreso promedio no constituyen buenas aproximaciones de los ingresos de las grandes mayorías. De hecho, el de las naciones mediante el IGM, más que el que se hace a partir del ingreso por habitante, es el que da lugar a diferencias considerables entre países. Por ejemplo, mientras que el ingreso nacional neto real por habitante en el año 2000 se encuentra $10 \%$ abajo del correspondiente a Estados Unidos, el ingreso real de la gran mayoría por habitante de Noruega resulta ser $4 \%$ más alto. Mucho mayor es el contraste que se observa entre México y Venezuela: el ingreso real por habitante de Venezuela es $6 \%$ menor que el de México, pero el IGM es $13 \%$ más alto. Nuestras estimaciones también nos permiten medir el ingreso por habitante de los quintiles superiores, lo que hemos llamado el ingreso de la minoría opulenta, y un resultado interesante es que los ingresos de los ricos son mucho más parecidos entre naciones que los ingresos de las grandes mayorías.

Un resultado que es particularmente sorprendente es que en cada nación el IGM es alrededor de 1.1 veces el ingreso nacional por habitante multiplicado por (1-Gini). Esta regla empírica es válida por igual para Dinamarca y Guatemala, así como para los otros 66 países en nuestra muestra. Se encuentra que una regla similar se ajusta para cualquier fracción de la población, con la única diferencia de que la constante cambia: así, se observa que el ingreso por habitante del primer $70 \%$ de la población en cualquier país es igual al PIB por habitante, descontado por la desigualdad del mismo país. Esta regla se muestra empíricamente, pero también se puede derivar teóricamente a partir de un enfoque econofísico de la distribución del ingreso.

A partir de estos resultados se pueden derivar dos conclusiones generales de política, así como una cuestión para investigación futura. Primero, es importante llevar a cabo comparaciones internacionales mediante el IGM, o alguna medida similar como el ingreso por habitante descontado por alguna medida de desigualdad, dado que estas medidas combinan adecuadamente los niveles de ingreso con los de desigualdad. Segundo, puesto que el ingreso por habitante de una parte de la población (con excepción de los muy ricos) depende directamente del ingreso por habitante y del factor (1-Gini), tanto el crecimiento económico como una mayor igualdad contribuyen por igual al mejoramiento de los niveles de bienestar. Hay que recordar que tanto las medidas impositivas como los subsidios pueden contribuir a ajustar la distribución del ingreso. Y lo anterior vuelve a plantear la eterna pregunta: ¿cuál es la relación entre crecimiento económico y 
cambios en la desigualdad? Nuestras medidas y resultados teóricos ofrecen una base para retomar este importante debate.

Finalmente, las comparaciones internacionales tienden a concentrase ya sea en los ingresos por habitante o en los ingresos de los más pobres (aquellos que disponen de menos de dos dólares para vivir). El indicador IGM agrega una nueva dimensión al combinar información sobre ingreso con la de su distribución para obtener una única medida del ingreso de la gran mayoría por habitante. Creemos que esto amplía la discusión sobre la desigualdad internacional y aclarará aspectos importantes en la bibliografía sobre desarrollo económico, como la que se refiere a la relación entre desigualdad y desarrollo, la liberalización comercial, género e inestabilidad política.

\section{REFERENCIAS BIBLIOGRÁFICAS}

Cowen, Tyler (2007), "Incomes and Inequality: What the Numbers Don't Tell Us," The New York Times, Nueva York.

Frumkin, Norman (2000), Guide to Economic Indicators, Armonk, M.E. Sharpe, Nueva York.

Kelley, Allen C. (1991), “The Human Development Index: Handle with Care”, Population and Development Review, 17 (2), pp. 315-324.

UNDP (1990), Human Development Report 1990, United Nations Development Programme, Nueva York. 
